\title{
Trapped modes around a row of circular cylinders in a channel
}

\author{
By T. UTSUNOMIYA ${ }^{1}$ AND R. EATOCK TAYLOR \\ ${ }^{1}$ Department of Civil Engineering, Kyoto University, Kyoto 606-8501, Japan \\ ${ }^{2}$ Department of Engineering Science, University of Oxford, Oxford, OX1 3PJ, UK
}

(Received 24 July 1998 and in revised form 14 December 1998)

Trapped modes around a row of bottom-mounted vertical circular cylinders in a channel are examined. The cylinders are identical, and their axes equally spaced in a plane perpendicular to the channel walls. The analysis has been made by employing the multipole expansion method under the assumption of linear water wave theory. At least the same number of trapped modes is shown to exist as the number of cylinders for both Neumann and Dirichlet trapped modes, with the exception that for cylinders having large radius the mode corresponding to the Dirichlet trapped mode for one cylinder will disappear. Close similarities between the Dirichlet trapped modes around a row of cylinders in a channel and the near-resonant phenomenon in the wave diffraction around a long array of cylinders in the open sea are discussed. An analogy with a mass-spring oscillating system is also presented.

\section{Introduction}

It has been reported by Maniar \& Newman (1997) that for large numbers of equally spaced, bottom-mounted vertical circular cylinders in line in the open sea, large wave forces will be excited on each of the cylinders at particular wavenumbers close to those of trapped modes. Similar observations have been made by Evans \& Porter (1997b) for circular arrays of bottom-mounted circular cylinders, and both of these cases may be understood as 'near-trapping'.

The existence of trapped modes for a bottom-mounted circular cylinder placed on the centreline of a wave channel was first established by Callan, Linton \& Evans (1991). It is now known that they may arise not only at wavenumbers below the cutoff value but also in the region above the cutoff value, or in the continuous spectrum. (The cutoff wavenumbers are $k=\pi / 2 d$ for a Neumann boundary condition applied on the channel walls, and $k=\pi / d$ for a Dirichlet boundary condition, both for antisymmetric waves with respect to the centreline of the channel, where $k$ is the wavenumber and $d$ is the half-width of the channel.) These cases include the trapped modes for a thin strip of finite length placed parallel to the walls but not on the centreline in the waveguide (Evans, Linton \& Ursell 1993), and those for a bottommounted circular cylinder placed on the centreline of the channel at the precise values of $a / d=0.3520905$ with $k=1.488884 \pi / d$ (for the Neumann trapped mode) and at $a / d=0.2670474$ with $k=1.991867 \pi / d$ (for the Dirichlet trapped mode) where $a$ is the radius of the cylinder (Evans \& Porter 1998). Here, we use the terminology 'Neumann trapped mode' for the trapped mode with a Neumann boundary condition being applied on the channel walls, and 'Dirichlet trapped mode' for a Dirichlet boundary 
condition being applied on them. In both cases a Neumann boundary condition is applied on each cylinder surface.

This paper first discusses other examples of trapped modes embedded in the continuous spectrum when $N$ bottom-mounted vertical circular cylinders having the same radius $a$ are equally spaced with their axes in the plane perpendicular to the channel walls in a channel. The distance between the centres of adjacent cylinders across the tank is taken to be $2 s$ and the distance between the channel wall and the centre of the cylinder at each end of the row is $s$.

Evans \& Porter (1997a) examined the trapped modes for multiple cylinders along the centreline of the channel, and observed the existence of up to $N$ trapped modes below the cutoff wavenumber. In this paper, we will show the existence of the same number of trapped modes as the number of cylinders across the tank, $N$, for both the Neumann trapped modes and the Dirichlet trapped modes, with the exception that for the latter, the highest trapped mode disappears when $a / s \gtrsim 0.677$ (Maniar \& Newman 1997; the figure was then modified to 0.6789 by Evans \& Porter 1997a). In both cases, the trapped modes, with the possible exception of the mode corresponding to the lowest frequency for the Dirichlet ones, are shown to be embedded in the continuous spectrum region.

Next, an analogy is given between the Dirichlet trapped modes for a row of equallyspaced cylinders in the channel and the near-resonant modes for cylinders in the open sea. Another analogy with a mass-spring oscillating system is also given, which may offer some insights into such resonant phenomena in wave diffraction problems.

\section{Formulation}

\subsection{Statement of the problem}

Cartesian coordinates $(x, y, z)$ are chosen such that the $(x, y)$-plane is located on the mean free surface and the $z$-axis points upward. The fluid is contained in a channel, the walls of which are located at $y= \pm d$ and $-\infty<x<\infty$, with the fluid depth being $h$. $N$ circular cylinders having the same radius $a$ and extending vertically throughout the fluid domain are located along the plane perpendicular to the channel walls, and without loss of generality the axes of the cylinders are positioned at $x=0$. The distance between the centres of adjacent cylinders is taken to be $2 s$ and the distance between the channel wall and the centre of the cylinder at each end of the row is $s$. Thus, the half-channel width $d$ is equal to Ns. The $(x, y)$ coordinates of the axis of each cylinder are represented by $\left(0, y_{j}\right)$, where

$$
y_{j}=-d+(2 j-1) s, \quad j=1,2, \ldots, N,
$$

and $N$ polar coordinates $\left(r_{j}, \theta_{j}\right)$ are defined with their origins at $\left(0, y_{j}\right)$ such that

$$
x=r_{j} \cos \theta_{j}, \quad y-y_{j}=r_{j} \sin \theta_{j}, \quad j=1,2, \ldots, N .
$$

Figure 1 illustrates the geometrical notation for the three-cylinder case $(N=3)$.

The fluid is assumed to be inviscid and incompressible, and its motion to be time harmonic with angular frequency $\omega$. Further, irrotational fluid motion is assumed so that a velocity potential $\Phi(x, y, z, t)$ exists. We employ the linearized boundary condition on the free surface $\left(\Phi_{z}=K \Phi\right.$ on $z=0$ where $K=\omega^{2} / g, g$ being the gravitational acceleration), and the rigid-wall condition on the bottom surface $\left(\Phi_{z}=0\right.$ on $\left.z=-h\right)$. By considering the configuration of the problem, the depth 


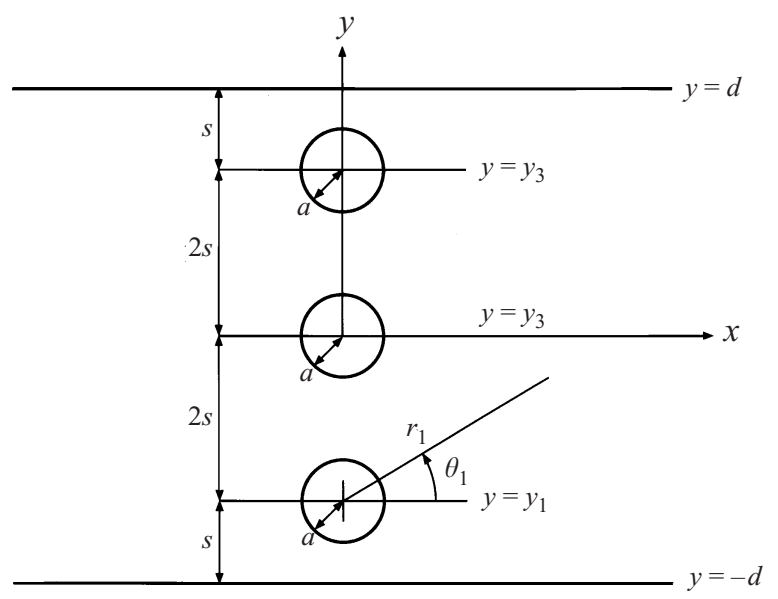

FIGURE 1. Geometrical notation of the problem for the three-cylinder case $(N=3)$.

dependence of the potential can be factored out as follows:

$$
\Phi(x, y, z, t)=\operatorname{Re}\left\{\phi(x, y) \frac{\cosh k(z+h)}{\cosh k h} \mathrm{e}^{-\mathrm{i} \omega t}\right\} .
$$

Here the wavenumber $k$ is related to the angular frequency $\omega$ by the dispersion relation

$$
\omega^{2}=g k \tanh k h .
$$

By substituting (2.3) into the Laplace equation, $\nabla^{2} \Phi(x, y, z, t)=0$, the Helmholtz equation is obtained as governing equation for the complex potential $\phi(x, y)$,

$$
\left(\nabla^{2}+k^{2}\right) \phi=0 \quad \text { in }|y|<d, \quad r_{j}>a, \quad j=1,2, \ldots, N .
$$

The boundary conditions for the Neumann trapped modes are

$$
\begin{gathered}
\phi_{y}=0 \quad \text { on }|y|=d, \quad-\infty<x<\infty, \\
\phi_{r_{j}}=0 \quad \text { on } r_{j}=a, \quad j=1,2, \ldots, N, \\
\phi \rightarrow 0 \quad \text { as }|x| \rightarrow \infty, \quad|y| \leqslant d .
\end{gathered}
$$

For the Dirichlet trapped modes, the boundary condition (2.6) should be replaced by

$$
\phi=0 \quad \text { on }|y|=d, \quad-\infty<x<\infty .
$$

Moreover, the symmetry of the trapped modes about the $y$-axis can be assumed in the problem considered herein (Callan et al. 1991), thus the following condition is also to be satisfied:

$$
\phi_{x}=0 \quad \text { on } x=0, \quad|y|<d, \quad\left|y-y_{j}\right|>a_{j}, \quad j=1,2, \ldots, N .
$$

\subsection{Multipole expansions}

We employ the so-called multipole expansion method to seek the trapped mode solutions. As the fundamental singular solutions which satisfy (2.5) and (2.10), we consider

$$
Y_{2 n}\left(k r_{j}\right) \cos 2 n \theta_{j} \quad \text { and } \quad Y_{2 n+1}\left(k r_{j}\right) \sin (2 n+1) \theta_{j} \text {, }
$$


where $Y_{n}$ denotes the Bessel function of the second kind and order $n$. For wave scattering problems, the fundamental singular solutions may be expressed by $H_{n}^{(1)}\left(k r_{j}\right) \cos n \theta_{j}$ and $H_{n}^{(1)}\left(k r_{j}\right) \sin n \theta_{j}$ as used by e.g. Linton \& Evans (1992) or McIver \& Bennett (1993), where $H_{n}^{(1)}$ denotes the Hankel function of the first kind and order $n$. Using only the imaginary part of the Hankel function (i.e. $Y_{n}$ ), however, will be more convenient than using the complex-valued function $H_{n}^{(1)}$, since we can then work only with real-valued functions in seeking the trapped mode solutions (see Evans \& Porter 1998). In the following, we modify the multipole expressions which have been derived by McIver $\&$ Bennett (1993) to suit the problem here.

The total potential $\phi(x, y)$ may be expressed in the following form:

$$
\phi=\sum_{j=1}^{N} \sum_{n=0}^{\infty}\left(A_{2 n}^{j} Z_{2 n} \phi_{2 n}^{j}+B_{2 n+1}^{j} Z_{2 n+1} \psi_{2 n+1}^{j}\right),
$$

where the factor $Z_{n}=J_{n}^{\prime}(k a) / Y_{n}^{\prime}(k a)$ is introduced for convenience, and $J_{n}$ denotes the Bessel function of the first kind and order $n$. In (2.12), $\phi_{2 n}^{j}$ and $\psi_{2 n+1}^{j}$ are channel multipoles that are singular at $\left(0, y_{j}\right)$ and satisfy $(2.5)$ and $(2.10)$ together with the boundary conditions on the channel walls, (2.6) or (2.9). They can be constructed from the integral representations of (2.11), with appropriate modification to include the boundary conditions on the channel walls. In the scattering problem or in the search for the trapped modes below cutoff values, each channel multipole may be constructed to satisfy appropriate radiation conditions or (2.8) at large distances $|x|$. In such cases, the remaining task is to determine the coefficients $A_{2 n}^{j}$ and $B_{2 n+1}^{j}$ to satisfy the boundary conditions on the cylinder surfaces.

As shown below, however, in this problem each multipole is generally accompanied by standing waves which do not vanish at large distances $|x|$, and so it does not satisfy (2.8) by itself. Therefore, in order to construct trapped mode solutions, we have to find the coefficients $A_{2 n}^{j}$ and $B_{2 n+1}^{j}$ such that the boundary condition (2.8) is satisfied together with the boundary conditions on the cylinder surfaces, (2.7).

Referring to (3.7) and (3.14) of McIver \& Bennett (1993) and modifying them to include the fundamental singular parts as in (2.11), we obtain the following channel multipoles:

$$
\begin{aligned}
\phi_{2 n}^{j}= & Y_{2 n}\left(k r_{j}\right) \cos 2 n \theta_{j} \\
& -\frac{(-1)^{n}}{2 \pi} f_{-\infty}^{\infty} \frac{\mathrm{e}^{k \gamma\left(y-y_{j}\right)}\left(\mathrm{e}^{-2 k \gamma d} \pm \mathrm{e}^{2 k \gamma y_{j}}\right)+\mathrm{e}^{-k \gamma\left(y-y_{j}\right)}\left(\mathrm{e}^{-2 k \gamma d} \pm \mathrm{e}^{-2 k \gamma y_{j}}\right)}{\gamma \sinh 2 k \gamma d} \\
& \times \cos k x t \cosh 2 n \tau \mathrm{d} t, \\
\psi_{2 n+1}^{j}= & Y_{2 n+1}\left(k r_{j}\right) \sin (2 n+1) \theta_{j} \\
& +\frac{(-1)^{n}}{2 \pi} f_{-\infty}^{\infty} \frac{\mathrm{e}^{k \gamma\left(y-y_{j}\right)}\left(\mathrm{e}^{-2 k \gamma d} \mp \mathrm{e}^{2 k \gamma y_{j}}\right)-\mathrm{e}^{-k \gamma\left(y-y_{j}\right)}\left(\mathrm{e}^{-2 k \gamma d} \mp \mathrm{e}^{-2 k \gamma y_{j}}\right)}{\gamma \sinh 2 k \gamma d} \\
& \times \cos k x t \sinh (2 n+1) \tau \mathrm{d} t,
\end{aligned}
$$

where

$$
t=\cosh \tau, \quad \gamma=\sinh \tau,
$$

and the upper and lower signs of \pm and $\mp$ correspond to Neumann and Dirichlet boundary condition, respectively, being satisfied on the channel walls. The integrals have to be evaluated as Cauchy principal value integrals, the physical meaning of which is that the behaviour of the channel multipoles at large distances $|x|$ represents 
a standing wave rather than a propagating wave, and by which the channel multipoles remain real-valued functions.

Further, modification of (3.12) and (3.16) and utilization of (3.5) in McIver \& Bennett (1993) leads to the following series expressions corresponding to (2.13) and (2.14):

$$
\begin{aligned}
\phi_{2 n}^{j}= & \frac{1}{k d} \sum_{m=0}^{M} \epsilon_{m}\left\{\begin{array}{c}
\cos (m \pi y / 2 d) \cos \left(m \pi y_{j} / 2 d\right) \\
\sin (m \pi y / 2 d) \sin \left(m \pi y_{j} / 2 d\right)
\end{array}\right\} t_{m}^{-1} \sin \left(k|x| t_{m}\right) c_{2 n}\left(t_{m}\right) \\
& -\frac{2}{k d} \sum_{m=M+1}^{\infty}\left\{\begin{array}{c}
\cos (m \pi y / 2 d) \cos \left(m \pi y_{j} / 2 d\right) \\
\sin (m \pi y / 2 d) \sin \left(m \pi y_{j} / 2 d\right)
\end{array}\right\} s_{m}^{-1} \mathrm{e}^{-k|x| s_{m}} d_{2 n}\left(s_{m}\right), \\
\psi_{2 n+1}^{j}= & \frac{2}{k d} \sum_{m=1}^{M}\left\{\begin{array}{c}
-\cos (m \pi y / 2 d) \sin \left(m \pi y_{j} / 2 d\right) \\
\sin (m \pi y / 2 d) \cos \left(m \pi y_{j} / 2 d\right)
\end{array}\right\} t_{m}^{-1} \sin \left(k|x| t_{m}\right) c_{2 n+1}\left(t_{m}\right) \\
& -\frac{2}{k d} \sum_{m=M+1}^{\infty}\left\{\begin{array}{c}
-\cos (m \pi y / 2 d) \sin \left(m \pi y_{j} / 2 d\right) \\
\sin (m \pi y / 2 d) \cos \left(m \pi y_{j} / 2 d\right)
\end{array}\right\} s_{m}^{-1} \mathrm{e}^{-k|x| s_{m}} d_{2 n+1}\left(s_{m}\right),
\end{aligned}
$$

where

$$
\begin{gathered}
t_{m}=\left(1-(m \pi / 2 k d)^{2}\right)^{1 / 2}, \\
s_{m}=\left((m \pi / 2 k d)^{2}-1\right)^{1 / 2}, \\
c_{n}\left(t_{m}\right)=\cos \left(n \sin ^{-1} t_{m}\right), \\
d_{n}\left(s_{m}\right)=\cosh \left(n \sinh ^{-1} s_{m}\right), \\
\epsilon_{0}=1, \quad \epsilon_{m}=2, \quad m=1,2, \ldots,
\end{gathered}
$$

and $M$ is an integer satisfying

$$
M \pi<2 k d<(M+1) \pi .
$$

In (2.16) and (2.17), for a Neumann boundary condition the upper expressions in the brackets should be taken for even values of $m$ and the lower expressions for odd values of $m$; whereas for a Dirichlet boundary condition the upper expressions should be taken for odd values of $m$ and the lower expressions for even values of $m$. This rule for the bracket will be retained throughout this paper.

As is obvious from (2.16) and (2.17), these multipole potentials satisfy the boundary conditions on the channel walls, (2.6) or (2.9). In general, however, they do not satisfy the boundary condition (2.8) because of the standing wave components which do not vanish at large distances $|x|$.

For a Neumann boundary condition $\psi_{2 n+1}^{j}$ does not include such a standing wave component and satisfies (2.8) when $k<\pi / 2 d(M=0)$. Those multipole potentials which vanish as $|x| \rightarrow \infty$ have been used as a basis for the construction of trapped modes in a channel enclosing a cylinder located on the centreline of the channel (Callan et al. 1991). Similarly, for a single cylinder located on the centreline of the channel $\left(y_{j}=0\right)$ with a Dirichlet boundary condition being applied on the channel walls, $\psi_{2 n+1}^{j}$ has no standing wave component when $k<\pi / d(M=1)$. The Dirichlet trapped mode around a single cylinder which is located on the centreline of the channel has been calculated by Maniar \& Newman (1997) by superposing such 
multipole potentials when $k<\pi / d$. It should be noted, however, that for a Dirichlet boundary condition both $\phi_{2 n}^{j}$ and $\psi_{2 n+1}^{j}$ have no standing wave component at large distances $|x|$ when $k<\pi / 2 d(M=0)$, which is consistent with the fact that the cutoff wavenumber for a Dirichlet boundary condition is $\pi / 2 d$ (as can be derived by the method of separation of variables at large distances $|x|)$.

In order to construct trapped modes using channel multipoles (2.13) and (2.14), those standing wave components which do not vanish as $|x| \rightarrow \infty$ must be cancelled out by appropriately choosing the coefficients $A_{2 n}^{j}$ and $B_{2 n+1}^{j}$. Now the trapped modes embedded in the continuous spectrum for one cylinder situated on the centreline of the channel can be constructed by appropriately choosing 'the strength of the Fourier components' $B_{2 n+1}$ (Evans \& Porter 1998). In this paper, instead, we form the trapped modes by appropriately choosing 'the strength of the multipoles radiated from each cylinder'; in other words, we adjust the coefficients $A_{2 n}^{j}$ and $B_{2 n+1}^{j}$ focusing on the $j$ index which represents cylinder number, in order to cancel out the standing wave components at large distances $|x|$.

Cancellation of the standing wave components is possible if we employ the following formulae. They were initially found heuristically in a preliminary numerical analysis of the problem by Utsunomiya \& Eatock Taylor (1998), but they are used here as an essential prerequisite to construct the trapped modes around a row of cylinders. This procedure leads to the major advantage as compared with our initial analysis that we can now avoid the use of complex arithmetic when searching for the trapped mode wavenumbers. Thus for Neumann trapped modes we use

$$
A_{2 n}^{j}=A_{2 n} \cos \left(\frac{(2 j-1) q \pi}{2 N}\right), \quad B_{2 n+1}^{j}=B_{2 n+1} \sin \left(\frac{(2 j-1) q \pi}{2 N}\right), \quad q=1,2, \ldots, N ;
$$

and for Dirichlet trapped modes we take

$$
\begin{gathered}
A_{2 n}^{j}=A_{2 n} \sin \left(\frac{(2 j-1) q \pi}{2 N}\right), \quad B_{2 n+1}^{j}=B_{2 n+1} \cos \left(\frac{(2 j-1) q \pi}{2 N}\right), \\
A_{2 n}^{j}=0, \quad B_{2 n+1}^{j}=B_{2 n+1}, \quad q=N,
\end{gathered}
$$

where we designate $q$ as the mode number of the trapped mode.

Inserting (2.16), (2.17) and (2.24) into (2.12), the following expression can be obtained for the Neumann trapped mode:

$$
\begin{aligned}
\phi_{N} \sim & \frac{1}{k d} \sum_{n=0}^{\infty}\left[A_{2 n} Z_{2 n} \sum_{m=0}^{M} \sum_{j=1}^{N} \epsilon_{m} \cos \left(\frac{(2 j-1) q \pi}{2 N}\right)\left\{\begin{array}{c}
\cos (m \pi y / 2 d) \cos \left(m \pi y_{j} / 2 d\right) \\
\sin (m \pi y / 2 d) \sin \left(m \pi y_{j} / 2 d\right)
\end{array}\right\}\right. \\
& \times t_{m}^{-1} \sin \left(k|x| t_{m}\right) c_{2 n}\left(t_{m}\right) \\
& +B_{2 n+1} Z_{2 n+1} \sum_{m=1}^{M} \sum_{j=1}^{N} 2 \sin \left(\frac{(2 j-1) q \pi}{2 N}\right)\left\{\begin{array}{r}
-\cos (m \pi y / 2 d) \sin \left(m \pi y_{j} / 2 d\right) \\
\sin (m \pi y / 2 d) \cos \left(m \pi y_{j} / 2 d\right)
\end{array}\right\} \\
& \left.\times t_{m}^{-1} \sin \left(k|x| t_{m}\right) c_{2 n+1}\left(t_{m}\right)\right], \quad|x| \rightarrow \infty,
\end{aligned}
$$

where subscript $N$ in $\phi_{N}$ specifies a Neumann trapped mode. Furthermore, using (2.1) and the formula (Gradshteyn \& Ryzhik 1994, 1.342)

$$
\sum_{j=1}^{n} \cos (2 j-1) x=\frac{1}{2} \sin 2 n x \operatorname{cosec} x
$$


we can obtain the relationships

$$
\begin{aligned}
\sum_{j=1}^{N} \cos & \left(\frac{(2 j-1) q \pi}{2 N}\right)\left\{\begin{array}{l}
\cos \left(m \pi y_{j} / 2 d\right) \\
\sin \left(m \pi y_{j} / 2 d\right)
\end{array}\right\} \\
& =\frac{1}{2}\left\{\begin{array}{l}
(-1)^{m / 2} \\
(-1)^{(m+1) / 2}
\end{array}\right\} \sum_{j=1}^{N}\left[\cos \left(\frac{(2 j-1)(m-q) \pi}{2 N}\right)+\cos \left(\frac{(2 j-1)(m+q) \pi}{2 N}\right)\right] \\
& \begin{cases}\neq 0 & (\text { for } m=q, 2 N-q, 2 N+q, 4 N-q, 4 N+q, \ldots) \\
=0 & \text { (otherwise) }\end{cases}
\end{aligned}
$$

and

$$
\begin{aligned}
& \sum_{j=1}^{N} \sin \left(\frac{(2 j-1) q \pi}{2 N}\right)\left\{\begin{array}{l}
\sin \left(m \pi y_{j} / 2 d\right) \\
\cos \left(m \pi y_{j} / 2 d\right)
\end{array}\right\} \\
& \quad=\frac{1}{2}\left\{\begin{array}{l}
(-1)^{m / 2} \\
(-1)^{(m-1) / 2}
\end{array}\right\} \sum_{j=1}^{N}\left[\cos \left(\frac{(2 j-1)(m-q) \pi}{2 N}\right)-\cos \left(\frac{(2 j-1)(m+q) \pi}{2 N}\right)\right] \\
& \begin{cases}\neq 0 & \text { (for } m=q, 2 N-q, 2 N+q, 4 N-q, 4 N+q, \ldots) \\
=0 & \text { (otherwise) }\end{cases}
\end{aligned}
$$

where for this case of a Neumann boundary condition the upper expressions in the brackets should be taken for even values of $m$ and the lower expressions for odd values of $m$.

Therefore, when $M<q$, the right-hand side of (2.27) vanishes, and thus the potential $\phi_{N}$ has no standing wave component at large distances $|x|$ and it satisfies the boundary condition (2.8). The condition $M<q$ is equivalent to $k<q \pi / 2 d$ $(q \geqslant M+1)$ from (2.23). Conversely when $k>q \pi / 2 d(q \leqslant M)$, the right-hand side of (2.27) does not vanish and the potential $\phi_{N}$ has standing wave components at large distances $|x|$. Therefore, the cutoff wavenumber for the problem in which the conditions (2.24) are applied becomes $q \pi / 2 d$ for $q=1,2, \ldots, N$.

Similarly, for Dirichlet trapped modes, we can show that the cutoff wavenumbers for the problems in which the conditions (2.25) or (2.26) are applied become $q \pi / 2 d$ for $q=1,2, \ldots, N-1$ or $q \pi / d$ for $q=N$, respectively. The derivation of the former cutoff wavenumber is almost the same as the case for Neumann trapped modes. The latter cutoff wavenumber $q \pi / d$ for $q=N$ can be derived as follows:

$$
\begin{aligned}
\phi_{D} \sim & \frac{2}{k d} \sum_{n=0}^{\infty} B_{2 n+1} Z_{2 n+1} \sum_{m=1}^{M} \sum_{j=1}^{N}\left\{\begin{array}{r}
-\cos (m \pi y / 2 d) \sin \left(m \pi y_{j} / 2 d\right) \\
\sin (m \pi y / 2 d) \cos \left(m \pi y_{j} / 2 d\right)
\end{array}\right\} \\
& \times t_{m}^{-1} \sin \left(k|x| t_{m}\right) c_{2 n+1}\left(t_{m}\right), \quad q=N,|x| \rightarrow \infty,
\end{aligned}
$$

where subscript $D$ in $\phi_{D}$ specifies a Dirichlet trapped mode. Furthermore, using (2.1) and (2.28) we can deduce that

$$
\begin{aligned}
\sum_{j=1}^{N}\left\{\begin{array}{l}
\sin \left(m \pi y_{j} / 2 d\right) \\
\cos \left(m \pi y_{j} / 2 d\right)
\end{array}\right\}= & \left\{\begin{array}{l}
(-1)^{(m+1) / 2} \\
(-1)^{m / 2}
\end{array}\right\} \sum_{j=1}^{N} \cos \left(\frac{(2 j-1) m \pi}{2 N}\right) \\
& \begin{cases}\neq 0 & \text { (for } m=2 N, 4 N, \ldots) \\
=0 & \text { (otherwise) }\end{cases}
\end{aligned}
$$

where for the case of a Dirichlet boundary condition the upper expressions in the 
brackets should be taken for odd values of $m$ and the lower expressions for even values of $m$. Therefore, when $M<2 N$, the right-hand side of (2.31) vanishes, and thus the potential $\phi_{D}$ has no standing wave component at large distances $|x|$ and it satisfies the boundary condition (2.8). The condition $M<2 N$ is equivalent to $k<N \pi / d(M+1 \leqslant 2 N)$ from $(2.23)$.

\subsection{Construction of trapped modes}

By the above procedure, the boundary conditions (2.6) (or (2.9)) and (2.8) together with (2.5) and (2.10) have already been satisfied by the total potential $\phi(x, y)$ defined by (2.12) when the relationships (2.24), (2.25) or (2.26) are applied. The remaining task for the construction of the trapped mode is to satisfy the boundary conditions on the cylinder surfaces (2.7). We can employ the same procedure as Linton \& McIver (1996) in the following to satisfy the boundary conditions on the multiple cylinder surfaces in the wave channel.

From (3.19)-(3.24) of McIver \& Bennett (1993),

$$
\begin{aligned}
\phi_{2 n}^{j}= & Y_{2 n}\left(k r_{j}\right) \cos 2 n \theta_{j} \\
& +\sum_{m=0}^{\infty}\left(\alpha_{2 n, 2 m}^{j} J_{2 m}\left(k r_{j}\right) \cos 2 m \theta_{j}+\beta_{2 n, 2 m+1}^{j} J_{2 m+1}\left(k r_{j}\right) \sin (2 m+1) \theta_{j}\right), \\
\psi_{2 n+1}^{j}= & Y_{2 n+1}\left(k r_{j}\right) \sin (2 n+1) \theta_{j} \\
& +\sum_{m=0}^{\infty}\left(a_{2 n+1,2 m}^{j} J_{2 m}\left(k r_{j}\right) \cos 2 m \theta_{j}+b_{2 n+1,2 m+1}^{j} J_{2 m+1}\left(k r_{j}\right) \sin (2 m+1) \theta_{j}\right),
\end{aligned}
$$

can be obtained, where the coefficients will be given later. Alternative expressions for the above multipoles, in which the singularity at $\left(0, y_{j}\right)$ is expanded about another point $\left(0, y_{p}\right), j \neq p$, can be obtained from (A.10)-(A.15) of Linton \& McIver (1996):

$$
\begin{aligned}
\phi_{2 n}^{j}= & \sum_{m=0}^{\infty}\left[\left(C_{2 n, 2 m}^{j p}+\alpha_{2 n, 2 m}^{j p}\right) J_{2 m}\left(k r_{p}\right) \cos 2 m \theta_{p}\right. \\
& \left.+\left(D_{2 n, 2 m+1}^{j p}+\beta_{2 n, 2 m+1}^{j p}\right) J_{2 m+1}\left(k r_{p}\right) \sin (2 m+1) \theta_{p}\right], \quad j \neq p ; \\
\psi_{2 n+1}^{j}= & \sum_{m=0}^{\infty}\left[\left(E_{2 n+1,2 m}^{j p}+a_{2 n+1,2 m}^{j p}\right) J_{2 m}\left(k r_{p}\right) \cos 2 m \theta_{p}\right. \\
& \left.+\left(F_{2 n+1,2 m+1}^{j p}+b_{2 n+1,2 m+1}^{j p}\right) J_{2 m+1}\left(k r_{p}\right) \sin (2 m+1) \theta_{p}\right], \quad j \neq p .
\end{aligned}
$$

The coefficients are as shown below, wherein only the imaginary parts of the coefficients given by (A.10)-(A.13) in Linton \& McIver (1996) are retained:

$$
\begin{aligned}
\alpha_{2 n, 2 m}^{j p}= & \frac{\epsilon_{m}(-1)^{n-m+1}}{\pi} f_{-\infty}^{\infty} \frac{\mathrm{e}^{-2 k \gamma d} \cosh k \gamma\left(y_{p}-y_{j}\right) \pm \cosh k \gamma\left(y_{p}+y_{j}\right)}{\gamma \sinh 2 k \gamma d} \\
& \times \cosh 2 m \tau \cosh 2 n \tau \mathrm{d} t, \\
\beta_{2 n, 2 m+1}^{j p}= & \frac{2(-1)^{n-m+1}}{\pi} f_{-\infty}^{\infty} \frac{\mathrm{e}^{-2 k \gamma d} \sinh k \gamma\left(y_{p}-y_{j}\right) \pm \sinh k \gamma\left(y_{p}+y_{j}\right)}{\gamma \sinh 2 k \gamma d} \\
& \times \sinh (2 m+1) \tau \cosh 2 n \tau \mathrm{d} t,
\end{aligned}
$$


Trapped modes around a row of cylinders in a channel

$$
\begin{aligned}
a_{2 n+1,2 m}^{j p}= & \frac{\epsilon_{m}(-1)^{n-m}}{\pi} f_{-\infty}^{\infty} \frac{\mathrm{e}^{-2 k \gamma d} \sinh k \gamma\left(y_{p}-y_{j}\right) \mp \sinh k \gamma\left(y_{p}+y_{j}\right)}{\gamma \sinh 2 k \gamma d} \\
& \times \cosh 2 m \tau \sinh (2 n+1) \tau \mathrm{d} t, \\
b_{2 n+1,2 m+1}^{j p}= & \frac{2(-1)^{n-m}}{\pi} f_{-\infty}^{\infty} \frac{\mathrm{e}^{-2 k \gamma d} \cosh k \gamma\left(y_{p}-y_{j}\right) \mp \cosh k \gamma\left(y_{p}+y_{j}\right)}{\gamma \sinh 2 k \gamma d} \\
& \times \sinh (2 m+1) \tau \sinh (2 n+1) \tau \mathrm{d} t,
\end{aligned}
$$

where the upper and lower signs of \pm and $\mp$ correspond to Neumann and Dirichlet boundary conditions respectively being satisfied on the channel walls. Also, the relationships $\alpha_{2 n, 2 m}^{j}=\alpha_{2 n, 2 m}^{j j}, \beta_{2 n, 2 m+1}^{j}=\beta_{2 n, 2 m+1}^{j j}, a_{2 n+1,2 m}^{j}=a_{2 n+1,2 m}^{j j}$ and $b_{2 n+1,2 m+1}^{j}=$ $b_{2 n+1,2 m+1}^{j j}$ hold. Moreover,

$$
\begin{gathered}
C_{2 n, 2 m}^{j p}=\frac{1}{2} \epsilon_{m}\left[(-1)^{m+n} Y_{2 n+2 m}\left(k R_{j p}\right)+(-1)^{m-n} Y_{2 n-2 m}\left(k R_{j p}\right)\right], \\
D_{2 n, 2 m+1}^{j p}= \pm\left[-(-1)^{m+n} Y_{2 n+2 m+1}\left(k R_{j p}\right)+(-1)^{m-n} Y_{2 n-2 m-1}\left(k R_{j p}\right)\right], \\
E_{2 n+1,2 m}^{j p}= \pm \frac{1}{2} \epsilon_{m}\left[(-1)^{m+n} Y_{2 n+2 m+1}\left(k R_{j p}\right)+(-1)^{m-n} Y_{2 n-2 m+1}\left(k R_{j p}\right)\right], \\
F_{2 n+1,2 m+1}^{j p}=-(-1)^{m+n} Y_{2 n+2 m+2}\left(k R_{j p}\right)+(-1)^{m-n} Y_{2 n-2 m}\left(k R_{j p}\right),
\end{gathered}
$$

where $R_{j p}=2 s|j-p|$ and the plus sign of \pm should be taken when $j<p$ and the minus sign when $j>p$.

Inserting the above multipoles (2.33)-(2.36) into (2.12), we can obtain the following expression for the total potential $\phi\left(r_{p}, \theta_{p}\right)$ in terms of the local polar coordinates $\left(r_{p}, \theta_{p}\right), p=1,2, \ldots, N$, which is valid only in the vicinity of the cylinder $p$ (the restriction arising from use of Graf's addition theorem in the derivation of (2.35) and $(2.36))$ :

$$
\begin{aligned}
\phi\left(r_{p}, \theta_{p}\right)= & \sum_{m=0}^{\infty}\left\{\tilde{A}_{2 m}^{p} Y_{2 m}\left(k r_{p}\right) \cos 2 m \theta_{p}+\tilde{B}_{2 m+1}^{p} Y_{2 m+1}\left(k r_{p}\right) \sin (2 m+1) \theta_{p}\right. \\
& +\sum_{j=1}^{N} \sum_{n=0}^{\infty}\left[\tilde{A}_{2 n}^{j}\left(\tilde{\alpha}_{2 n, 2 m}^{j p} J_{2 m}\left(k r_{p}\right) \cos 2 m \theta_{p}+\tilde{\beta}_{2 n, 2 m+1}^{j p} J_{2 m+1}\left(k r_{p}\right) \sin (2 m+1) \theta_{p}\right)\right. \\
& \left.\left.+\tilde{B}_{2 n+1}^{j}\left(\tilde{a}_{2 n+1,2 m}^{j p} J_{2 m}\left(k r_{p}\right) \cos 2 m \theta_{p}+\tilde{b}_{2 n+1,2 m+1}^{j p} J_{2 m+1}\left(k r_{p}\right) \sin (2 m+1) \theta_{p}\right)\right]\right\} .
\end{aligned}
$$

Here

and

$$
\tilde{A}_{2 n}^{j}=Z_{2 n} A_{2 n}^{j}, \quad \tilde{B}_{2 n+1}^{j}=Z_{2 n+1} B_{2 n+1}^{j},
$$

$$
\tilde{\alpha}_{2 n, 2 m}^{j p}= \begin{cases}C_{2 n, 2 m}^{j p}+\alpha_{2 n, 2 m}^{j p} & (\text { for } j \neq p) \\ \alpha_{2 n, 2 m}^{p p} & (\text { for } j=p),\end{cases}
$$

are used for convenience. The coefficients $\tilde{\beta}_{2 n, 2 m+1}^{j p}, \tilde{a}_{2 n+1,2 m}^{j p}$ and $\tilde{b}_{2 n+1,2 m+1}^{j p}$ are similarly defined as above.

Applying the boundary conditions (2.7) on each of the cylinder surfaces, $p=$ $1,2, \ldots, N$, we finally obtain the following homogeneous systems of equations:

$$
A_{2 m}^{p}+\sum_{j=1}^{N} \sum_{n=0}^{\infty}\left(\tilde{A}_{2 n}^{j} \tilde{\alpha}_{2 n, 2 m}^{j p}+\tilde{B}_{2 n+1}^{j} \tilde{a}_{2 n+1,2 m}^{j p}\right)=0,
$$




$$
B_{2 m+1}^{p}+\sum_{j=1}^{N} \sum_{n=0}^{\infty}\left(\tilde{A}_{2 n}^{j} \tilde{\beta}_{2 n, 2 m+1}^{j p}+\tilde{B}_{2 n+1}^{j} \tilde{b}_{2 n+1,2 m+1}^{j p}\right)=0,
$$

where $p=1,2, \ldots, N$ and $m=0,1, \ldots, \infty$ in both cases. In the numerical calculations, the infinite systems must be truncated in terms of the Fourier modes, $n$ and $m$, and the total number of Fourier modes is defined by $N_{F}$ both for odd and even modes.

Application of the relationships (2.24), (2.25) or (2.26) to the truncated systems of equations (2.48) and (2.49) leads to the following homogeneous systems of equations: for Neumann trapped modes corresponding to $q=1,2, \ldots, N-1$

$$
\begin{aligned}
A_{2 m} \cos \left(\frac{(2 p-1) q \pi}{2 N}\right)+ & \sum_{n=0}^{N_{F}-1}\left[\tilde{A}_{2 n} \sum_{j=1}^{N} \cos \left(\frac{(2 j-1) q \pi}{2 N}\right) \tilde{\alpha}_{2 n, 2 m}^{j p}\right. \\
& \left.+\tilde{B}_{2 n+1} \sum_{j=1}^{N} \sin \left(\frac{(2 j-1) q \pi}{2 N}\right) \tilde{a}_{2 n+1,2 m}^{j p}\right]=0, \\
B_{2 m+1} \sin \left(\frac{(2 p-1) q \pi}{2 N}\right)+ & \sum_{n=0}^{N_{F}-1}\left[\tilde{A}_{2 n} \sum_{j=1}^{N} \cos \left(\frac{(2 j-1) q \pi}{2 N}\right) \tilde{\beta}_{2 n, 2 m+1}^{j p}\right. \\
& \left.+\tilde{B}_{2 n+1} \sum_{j=1}^{N} \sin \left(\frac{(2 j-1) q \pi}{2 N}\right) \tilde{b}_{2 n+1,2 m+1}^{j p}\right]=0 ;
\end{aligned}
$$

and for the Neumann trapped mode corresponding to $q=N$

$$
B_{2 m+1}(-1)^{p-1}+\sum_{n=0}^{N_{F}-1} \tilde{B}_{2 n+1} \sum_{j=1}^{N}(-1)^{j-1} \tilde{b}_{2 n+1,2 m+1}^{j p}=0 .
$$

Similarly, for Dirichlet trapped modes corresponding to $q=1,2, \ldots, N-1$ :

$$
\begin{aligned}
A_{2 m} \sin \left(\frac{(2 p-1) q \pi}{2 N}\right) & +\sum_{n=0}^{N_{F}-1}\left[\tilde{A}_{2 n} \sum_{j=1}^{N} \sin \left(\frac{(2 j-1) q \pi}{2 N}\right) \tilde{\alpha}_{2 n, 2 m}^{j p}\right. \\
& \left.+\tilde{B}_{2 n+1} \sum_{j=1}^{N} \cos \left(\frac{(2 j-1) q \pi}{2 N}\right) \tilde{a}_{2 n+1,2 m}^{j p}\right]=0, \\
B_{2 m+1} \cos \left(\frac{(2 p-1) q \pi}{2 N}\right)+ & \sum_{n=0}^{N_{F}-1}\left[\tilde{A}_{2 n} \sum_{j=1}^{N} \sin \left(\frac{(2 j-1) q \pi}{2 N}\right) \tilde{\beta}_{2 n, 2 m+1}^{j p}\right. \\
& \left.+\tilde{B}_{2 n+1} \sum_{j=1}^{N} \cos \left(\frac{(2 j-1) q \pi}{2 N}\right) \tilde{b}_{2 n+1,2 m+1}^{j p}\right]=0 ;
\end{aligned}
$$

and for the Dirichlet trapped mode corresponding to $q=N$

$$
B_{2 m+1}+\sum_{n=0}^{N_{F}-1} \tilde{B}_{2 n+1} \sum_{j=1}^{N} \tilde{b}_{2 n+1,2 m+1}^{j p}=0 .
$$

Non-trivial solutions of the above homogeneous systems of equations will exist if and only if the determinant of the real coefficient matrix vanishes. If such a 


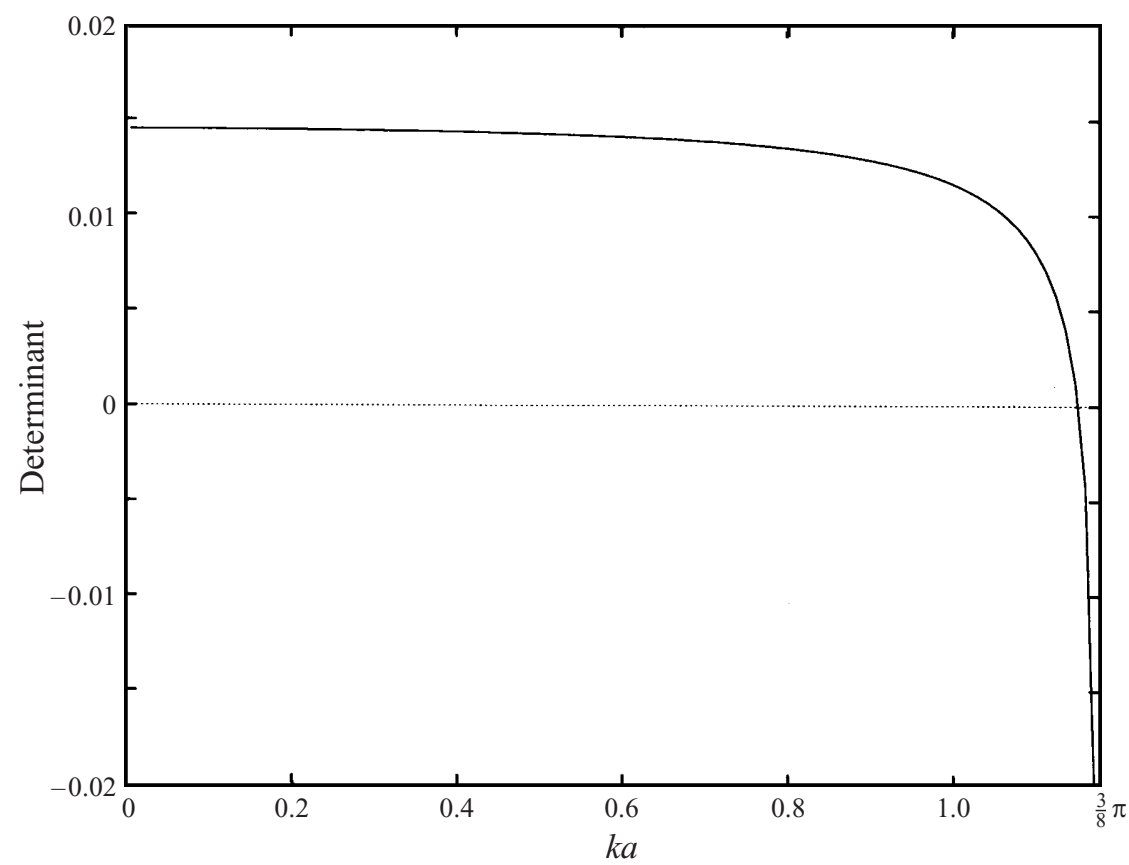

FIGURE 2. Determinant of the system matrix versus wavenumber for $q=3, N=4$, and $N_{F}=4$ in the Neumann channel $(a / s=0.5)$.

vanishing point can be found, it will represent the trapped mode wavenumber. In the next section, we calculate the determinant of the $2 N_{F} \times 2 N_{F}$ system matrix for $q=1,2, \ldots, N-1$ and that of $N_{F} \times N_{F}$ system matrix for $q=N$, and search for the determinant's vanishing point by numerical analysis.

\section{Results}

\subsection{Trapped modes in a channel}

In the numerical evaluation of the coefficient matrix, the infinite integrals (2.37)-(2.40) must be evaluated with high accuracy. Here the method described in Appendix A of McIver \& Bennett (1993) has been implemented, and satisfactory results have been obtained as shown below. The determinant of the matrix has been computed by utilizing the LU decomposition. The search for the determinant's vanishing point has been made by using the NAG library subroutine c05adf, which uses Brent's method. An example of the variation of the determinant with wavenumber is shown in figure 2 . As is obvious, for each mode the determinant changes its sign only once below the cutoff wavenumber; thus the trapped mode wavenumber can be found easily by specifying two points in Brent's method such that the determinant has opposite signs.

In order to examine the effect of truncation of the Fourier modes, Neumann trapped mode wavenumbers have been calculated for various values of $N_{F}$ and the results are shown in table 1 for $a / s=0.5$ and $a / s=1.0$. The latter spacing is the special case which corresponds to the cylinders touching, and creating a barrier across the tank. Only one trapped mode wavenumber has been obtained for each mode $q$, just below the corresponding cutoff wavenumber, $k s=q \pi / 2 N$ (or $k=q \pi / 2 d$ ). For 


\begin{tabular}{cccccccc}
\hline & $N$ & $q$ & $N_{F}=1$ & $N_{F}=2$ & $N_{F}=4$ & $N_{F}=8$ & $N_{F}=16$ \\
$(a) \quad 4$ & 1 & 0.392169 & 0.392164 & 0.392164 & 0.392164 & 0.392164 \\
& 4 & 2 & 0.780408 & 0.780278 & 0.780278 & 0.780278 & 0.780278 \\
& 4 & 3 & 1.152666 & 1.151933 & 1.151931 & 1.151931 & 1.151931 \\
& 4 & 4 & 1.391316 & 1.391314 & 1.391313 & 1.391313 & 1.391313 \\
& 1 & 1 & 1.391316 & 1.391314 & 1.391314 & 1.391314 & - \\
$(b) \quad 5$ & 1 & 0.314062 & 0.314010 & 0.314007 & 0.314007 & 0.314007 \\
& 5 & 2 & 0.627376 & 0.626983 & 0.626938 & 0.626938 & 0.626938 \\
5 & 3 & 0.938123 & 0.936807 & 0.936507 & 0.936505 & 0.936505 \\
& 5 & 4 & 1.238101 & 1.234641 & 1.233664 & 1.233661 & 1.233661 \\
5 & 5 & 1.430229 & 1.427529 & 1.427480 & 1.427480 & 1.427480 \\
TABLE 1. Neumann trapped mode wavenumber $k s$ for $(a) N=4$ and $a / s=0.5$, and $(b) N=5$ \\
and $a / s=1.0$ together with the computations of Maniar \& Newman $(1997)$ for a single cylinder \\
$(N=q=1)$.
\end{tabular}

the case of $N=4$ and $a / s=0.5,(a)$, six-figure accuracy after the decimal point is attained with the number of Fourier modes $N_{F}=4$. For the case of $N=5$ and $a / s=1.0,(b)$, six-figure accuracy after the decimal point is attained with $N_{F}=8$, and the results for $N_{F}=4$ give five-figure accuracy after the decimal point. Comparisons with Neumann trapped mode wavenumbers for the single cylinder calculated by Maniar \& Newman (1997) are also made, and satisfactory agreement for the cases of $N=q=4$ and $N=q=5$ is observed. In the subsequent calculations we generally employ $N_{F}=8$ and retain six figures after the decimal point; but for cases with large numbers of cylinders we employ $N_{F}=4$ and reduce the significant number of figures after the decimal point to five. This is based on consideration of the computational effort (the most time consuming part of the computation is for evaluation of infinite integrals (2.37)-(2.40), and the computation time is almost proportional to $N \times N_{F}^{2}$ ).

Table 2 shows the Neumann trapped mode wavenumbers $(a)$ and Dirichlet trapped mode numbers $(b)$ both for $a / s=0.5$ and for different numbers of cylinders. For each value of $q$, only one vanishing point of the determinant has been found. Therefore, we have obtained $N$ trapped modes for $N$ cylinders in the case of $a / s=0.5$.

Figure 3 shows the equipotential contours of the Neumann trapped mode waves for the case of four cylinders $(N=4)$ and $a / s=0.5$. Because of the symmetry of the trapped waves, only one quadrant of the full channel is shown.

The highest $(N$ th) trapped mode in each case is equivalent to that for the case of one cylinder, as seen from figure 3 and table 2; actually we can generate the trapped mode potential by appropriately copying the trapped mode potential for the one cylinder case. For the Neumann trapped mode,

$$
\left.\begin{array}{cc}
\phi_{N}\left(x, y-y_{1}\right)=\bar{\phi}_{N}(x, y), & \left|y-y_{1}\right| \leqslant s \\
\phi_{N}\left(x, y-y_{2}\right)=-\bar{\phi}_{N}(x, y), & \left|y-y_{2}\right| \leqslant s \\
\phi_{N}\left(x, y-y_{3}\right)=\bar{\phi}_{N}(x, y), & \left|y-y_{3}\right| \leqslant s \\
\cdots, &
\end{array}\right\}
$$

where $\bar{\phi}_{N}(x, y)$ is the Neumann trapped mode potential for the one cylinder case. 
(a)

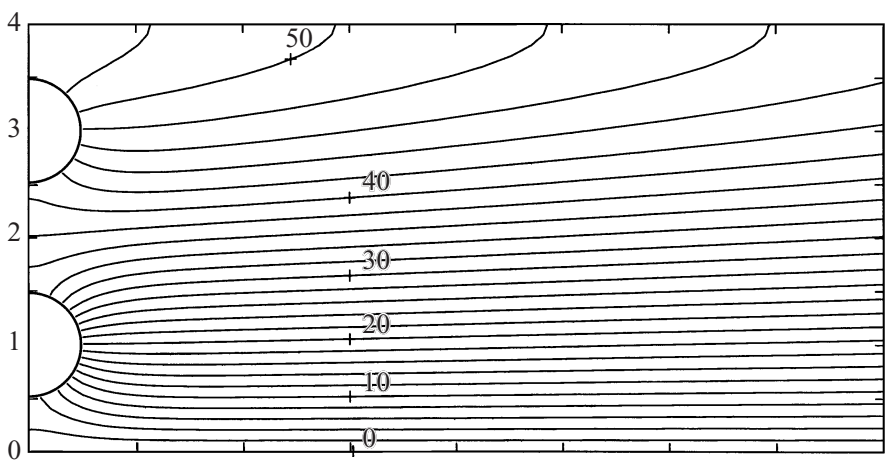

(b)

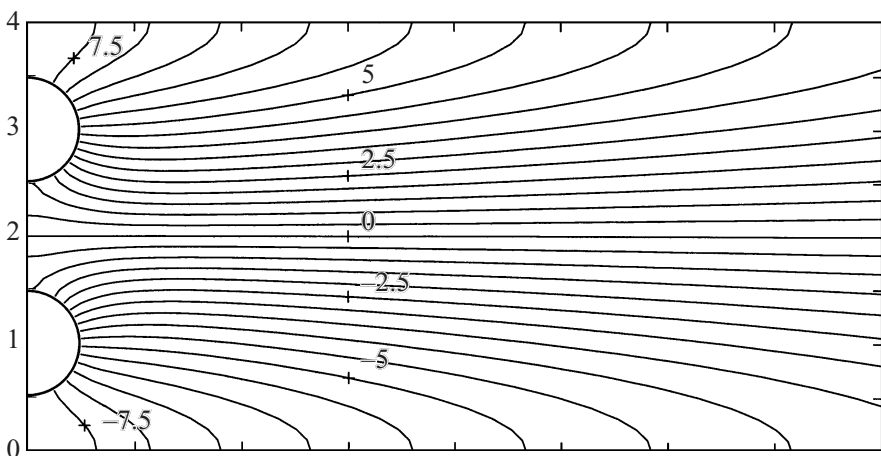

(c)

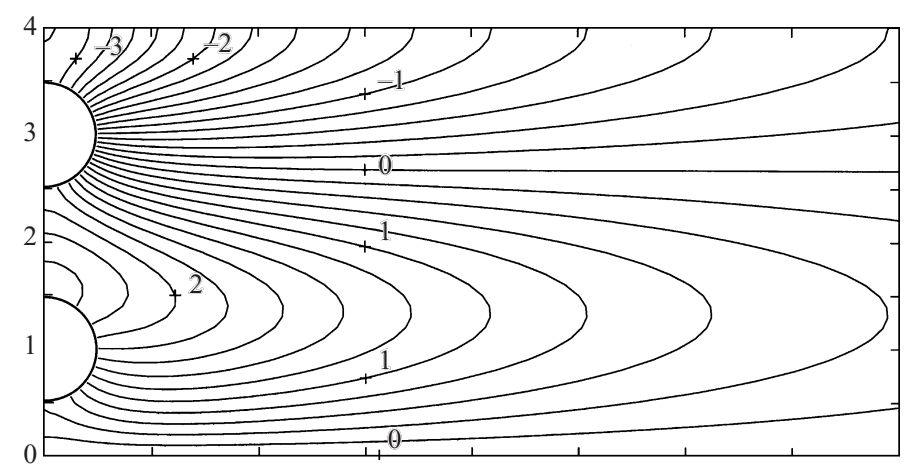

(d)

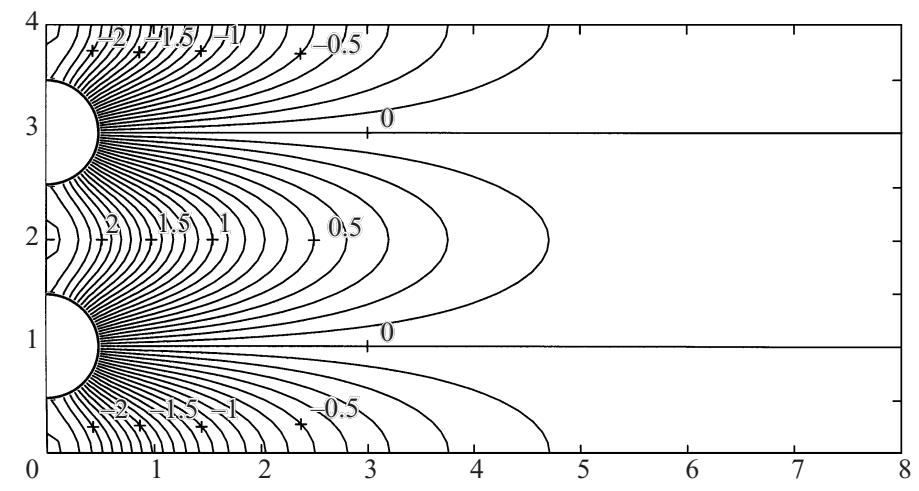

FigURE 3. Equipotential contours of the Neumann trapped mode waves for $N=4$ and $a / s=0.5$ : (a) $q=1$, (b) $q=2$, (c) $q=3$ and (d) $q=4$. 


\begin{tabular}{cccccccccc}
\hline & & & & & & & & & \\
& & $q=1$ & $q=2$ & $q=3$ & $q=4$ & $q=5$ & $q=6$ & $q=7$ & $q=8$ \\
(a) & 1 & 1.391313 & - & - & - & - & - & - & - \\
& 2 & 0.780278 & 1.391313 & - & - & - & - & - & - \\
& 3 & 0.522275 & 1.031837 & 1.391313 & - & - & - & - & - \\
& 4 & 0.392164 & 0.780278 & 1.151931 & 1.391313 & - & - & - & - \\
& 5 & 0.313891 & 0.625925 & 0.932446 & 1.220160 & 1.391313 & - & - & - \\
& 6 & 0.261646 & 0.522275 & 0.780278 & 1.031837 & 1.262991 & 1.391313 & - & - \\
& 7 & 0.224303 & 0.447987 & 0.670186 & 0.889272 & 1.101208 & 1.291705 & 1.391313 & - \\
& 8 & 0.196285 & 0.392164 & 0.587112 & 0.780278 & 0.969965 & 1.151931 & 1.311888 & 1.391313 \\
(b) & 1 & 3.071722 & - & - & - & - & - & - & - \\
& 2 & 0.780278 & 3.071722 & - & - & - & - & - & - \\
& 3 & 0.522275 & 1.031837 & 3.071722 & - & - & - & - & - \\
4 & 0.392164 & 0.780278 & 1.151931 & 3.071722 & - & - & - & - \\
5 & 0.313891 & 0.625925 & 0.932446 & 1.220160 & 3.071722 & - & - & - \\
6 & 0.261646 & 0.522275 & 0.780278 & 1.031837 & 1.262991 & 3.071722 & - & - \\
7 & 0.224303 & 0.447987 & 0.670186 & 0.889272 & 1.101208 & 1.291706 & 3.071722 & - \\
8 & 0.196285 & 0.392164 & 0.587112 & 0.780278 & 0.969965 & 1.151931 & 1.311888 & 3.071722
\end{tabular}

TABLE 2. Neumann trapped mode wavenumber $k s(a)$ and Dirichlet trapped mode wavenumber $k s$ (b), both for $a / s=0.5$ and $N_{F}=8$.

Similarly for the Dirichlet mode,

$$
\left.\begin{array}{cc}
\phi_{D}\left(x, y-y_{1}\right)=\bar{\phi}_{D}(x, y), & \left|y-y_{1}\right| \leqslant s, \\
\phi_{D}\left(x, y-y_{2}\right)=\bar{\phi}_{D}(x, y), & \left|y-y_{2}\right| \leqslant s \\
\phi_{D}\left(x, y-y_{3}\right)=\bar{\phi}_{D}(x, y), & \left|y-y_{3}\right| \leqslant s, \\
\cdots, &
\end{array}\right\}
$$

where $\bar{\phi}_{D}(x, y)$ is the Dirichlet trapped mode potential for the one cylinder case. These potentials satisfy all of the boundary conditions (2.6) (or (2.9)), (2.7) and (2.8) for the trapped mode together with the governing equation (2.5) and the symmetry condition (2.10); they thus represent trapped modes. Obviously, these solutions correspond to the $N$ th trapped mode in table 2.

It may also be noted from figure 3 and also from table 2 that the second mode for four cylinders is identical to the first mode for two cylinders: the same trapped mode wavenumbers are obtained for the case of $N=4$ and $q=2$ and that of $N=2$ and $q=1$. Similar relationships may be found between the trapped modes for three cylinders and those for six cylinders or between four and eight cylinders. More generally the same trapped mode wavenumber is obtained for the same value of $q / N$ (and for the same radius of cylinders; this is apparent from table 1). Such relationships can be justified by expressions similar to (3.1) or (3.2), by appropriately copying the trapped mode potential for the same value of $q / N$.

Another interesting feature observed in table 2 is that the same trapped mode wavenumbers are obtained for the Dirichlet and Neumann trapped modes at the same values of $q$ and $N$, except for the $N$ th mode. We could anticipate an $N$ th Dirichlet trapped mode at $k s=1.391313$ for this case, considering the symmetry between $(a)$ and $(b)$ of table 2. However, non-existence of such a trapped mode is consistent with the mathematical proof given by McIver \& Linton (1995): they proved the non-existence of the Dirichlet trapped mode below $k s<\pi / 2$ for an obstacle satisfying $n_{y} \leqslant 0$ if $y \geqslant 0$, and $n_{y} \geqslant 0$ if $y \leqslant 0$ (where $\left(n_{x}, n_{y}\right)$ is the normal 
to the obstacle out of the fluid region). In (2.25) the condition for $q=N$ has been excluded in advance; this corresponds to the anticipated Dirichlet trapped mode (at $k s=1.381313$ for this case), which in fact does not exist.

The reason why the same trapped wavenumber is obtained in the Neumann and Dirichlet modes at the same values of $N$ and $q$ (except when $N=q$ ) may be explained in a similar way to the argument based on (3.1) and (3.2) when $N$ is an even number. For example when $N=2$ and $q=1$, the Dirichlet trapped mode potential $\phi_{D}(x, y)$ can be generated from the Neumann trapped mode potential $\phi_{N}(x, y)$ by

$$
\left.\begin{array}{ll}
\phi_{D}(x, y)=-\phi_{N}(x, d-y), & y \geqslant 0, \\
\phi_{D}(x, y)=\phi_{N}(x,-d-y), & y \leqslant 0 .
\end{array}\right\}
$$

Similar construction of the equivalent trapped mode potentials is always possible when $N$ is an even number. However, when $N$ is an odd number, e.g. when $N=3$ and $q=1$, such a simple method to generate equivalent trapped mode potentials in the Neumann and Dirichlet modes seems not to exist.

The connection with the cutoff wavenumber (frequency) is now examined. As derived by the method of separation of variables, the cutoff wavenumber of the channel is 0 for Neumann trapped modes and $k=\pi / 2 d$ for Dirichlet trapped modes. Thus, excluding the single cylinder case $(N=1)$, we see that only the first $(q=1)$ Dirichlet trapped modes are below the cutoff wavenumber of $k=\pi / 2 d(k s=\pi / 2 N$ if $d=N s$ is applied), and otherwise the modes are all above the cutoff. In other words, they are embedded in the continuous spectrum.

If we restrict ourselves to antisymmetric waves with respect to the centreline of the channel, the cutoff wavenumber becomes $k=\pi / 2 d(k s=\pi / 2 N)$ for Neumann trapped modes and $k=\pi / d(k s=\pi / N)$ for Dirichlet trapped modes. For such cutoff wavenumbers, the first $(q=1)$ Neumann trapped modes are included below the cutoff, and the second $(q=2)$ Dirichlet trapped modes are included below the cutoff, excluding the case $N=2$.

In the process of the construction of trapped modes, however, we enforced the relationships (2.24), (2.25) or (2.26) and eliminated the standing (or propagating) wave components at large $|x|$. For such modified problems, the cutoff wavenumbers are considered to be $k=q \pi / 2 d(k s=q \pi / 2 N)$ for Neumann and Dirichlet modes, except for the case $k=N \pi / d(k s=\pi)$ for the $N$ th Dirichlet mode. In our investigation the vanishing points of the determinant have been sought below the corresponding cutoff wavenumbers, and only one vanishing point has been found for each case, just below the cutoff wavenumber. We might find trapped modes even above the cutoff wavenumbers, using a method similar to that of Evans \& Porter (1998), but that is beyond the scope of this paper.

As stated above, the cutoff wavenumber varies depending on how we pose the problem (see Evans, Levitin \& Vassiliev 1994). It may however be reasonable to conclude that these trapped modes on multiple cylinders are above the cutoff wavenumber (with the possible exception of the first Dirichlet trapped modes), since each multipole potential radiates standing (propagating) waves at large distances $|x|$, and cancellation of these has been enforced by means of special relationships for the coefficients $A_{2 n}^{j}$ and $B_{2 n+1}^{j}$.

Table 3 shows (a) the Neumann and (b) Dirichlet trapped mode wavenumbers $k s$ for four cylinders and various values of the cylinder radius $a / s$. For every case of the Neumann mode, four trapped modes are obtained. On the other hand, for the Dirichlet mode the highest mode disappears as the cylinder radius becomes large. 


\begin{tabular}{ccccccc}
\hline & & & & & & \\
(a) & & $q=1$ & $q=2$ & $q=3$ & $q=4$ & $N=q=1$ \\
& 0.1 & 0.392697 & 0.785383 & 1.178043 & 1.569050 & 1.569051 \\
& 0.2 & 0.392672 & 0.785167 & 1.177154 & 1.550230 & 1.550230 \\
& 0.3 & 0.392583 & 0.784351 & 1.173315 & 1.504842 & 1.504842 \\
& 0.4 & 0.392405 & 0.782655 & 1.164617 & 1.446547 & 1.446547 \\
& 0.5 & 0.392164 & 0.780278 & 1.151931 & 1.391313 & 1.391314 \\
& 0.6 & 0.391317 & 0.777932 & 1.139058 & 1.348300 & 1.348300 \\
& 0.7 & 0.391817 & 0.776556 & 1.130706 & 1.322882 & 1.322882 \\
& 0.8 & 0.391867 & 0.776899 & 1.131026 & 1.320790 & 1.320789 \\
& 0.9 & 0.392086 & 0.779121 & 1.142527 & 1.351785 & 1.351785 \\
& 1.0 & 0.392395 & 0.782404 & 1.161919 & 1.427481 & 1.427481 \\
& 0.1 & 0.392697 & 0.785383 & 1.178043 & 3.129605 & 3.129605 \\
& 0.2 & 0.392672 & 0.785167 & 1.177154 & 3.055177 & 3.055177 \\
& 0.3 & 0.392583 & 0.784351 & 1.173315 & 2.989795 & 2.989795 \\
& 0.4 & 0.392405 & 0.782655 & 1.164617 & 2.999586 & 2.999586 \\
& 0.5 & 0.392164 & 0.780278 & 1.151931 & 3.071722 & 3.071722 \\
& 0.6 & 0.391317 & 0.777932 & 1.139057 & 3.132556 & 3.132556 \\
& 0.7 & 0.391817 & 0.776556 & 1.130706 & - & - \\
& 0.8 & 0.391867 & 0.776899 & 1.131026 & - & - \\
& 0.9 & 0.392086 & 0.779121 & 1.142528 & - & - \\
& 1.0 & 0.392395 & 0.782404 & 1.161919 & - & -
\end{tabular}

TABLE 3. Neumann trapped mode wavenumber $k s(a)$ and Dirichlet trapped mode wavenumber $k s$ (b) both for $N=4$ and $N_{F}=8$, together with the computations of Maniar \& Newman (1997) for a single cylinder $(N=q=1)$.

As a check on the numerical accuracy, the trapped mode wavenumbers for one cylinder $(N=q=1)$ are also indicated, figures for which are obtained from Maniar $\&$ Newman (1997). The trapped mode wavenumbers for one cylinder by the program developed here are exactly the same as the $q=4$ column of table 3 to sevenfigure accuracy. The agreement between the $q=4$ column and the trapped mode wavenumber for one cylinder shows the equivalency between the two cases, together with the satisfactory numerical accuracy.

As is already known, the Dirichlet trapped mode for one cylinder only exists when $a / s \lesssim 0.6789$ (Maniar \& Newman 1997; Evans \& Porter 1997). Because of the equivalency of the $N$ th trapped mode with the trapped mode for a single cylinder, the $N$ th Dirichlet trapped mode disappears at large values of $a / s(\gtrsim 0.6789)$ as expected.

We may conclude therefore that at least $N$ trapped modes exist for an array of $N$ equally-spaced circular cylinders of the same radius in a channel, with the exception that the trapped mode corresponding to the Dirichlet one for a single cylinder disappears for large radius cylinders $(a / s \gtrsim 0.6789)$. We cannot remove the word 'at least' in the above arguments, because the construction of the trapped mode here is based on (2.24), (2.25) or (2.26), which are only sufficient conditions to satisfy (2.8) and not necessary conditions. Actually, if we consider the cylinder array for $a / s=0.3520905$, the array should have at least one additional Neumann trapped mode at $k s=1.488884 \pi$ (Evans \& Porter 1998) and the number of trapped modes will become at least $N+1$ in such a case.

\subsection{Analogy with the near-resonant phenomenon around a long array of cylinders}

The near-resonant phenomenon around a long array of cylinders in the open sea presented by Maniar \& Newman (1997) will be considered next, and related to the trapped modes around a row of cylinders in a channel. 
The wave force induced on each cylinder of the array in a channel can be deduced by using the same procedure as Linton \& McIver (1996). Inserting (2.48) and (2.49) into (2.45), we obtain

$$
\begin{aligned}
\phi\left(r_{j}, \theta_{j}\right)= & \sum_{m=0}^{\infty}\left\{A_{2 m}^{j}\left(Z_{2 m} Y_{2 m}\left(k r_{j}\right)-J_{2 m}\left(k r_{j}\right)\right) \cos 2 m \theta_{j}\right. \\
& \left.+B_{2 m+1}^{j}\left(Z_{2 m+1} Y_{2 m+1}\left(k r_{j}\right)-J_{2 m+1}\left(k r_{j}\right)\right) \sin (2 m+1) \theta_{j}\right\} ;
\end{aligned}
$$

and using the Wronskian relations for Bessel functions in the expressions on the cylinder surfaces, we have

$$
\phi\left(a, \theta_{j}\right)=-\frac{2}{\pi k a} \sum_{m=0}^{\infty}\left\{\frac{A_{2 m}^{j} \cos 2 m \theta_{j}}{Y_{2 m}^{\prime}(k a)}+\frac{B_{2 m+1}^{j} \sin (2 m+1) \theta_{j}}{Y_{2 m+1}^{\prime}(k a)}\right\} .
$$

By utilizing (2.3) and integrating the pressure on the cylinder surface, the wave force on the $j$ th cylinder in the $y$-direction can be expressed as $\operatorname{Re}\left\{Y_{j} \mathrm{e}^{-\mathrm{i} \omega t}\right\}$ where

$$
Y_{j}=-\frac{\rho \mathrm{i} a \omega \tanh k h}{k} \int_{0}^{2 \pi} \phi\left(a, \theta_{j}\right) \sin \theta_{j} \mathrm{~d} \theta_{j}=\frac{2 \rho \mathrm{i} \omega \tanh k h}{k^{2} Y_{1}^{\prime}(k a)} B_{1}^{j},
$$

and $\rho$ is the density of the fluid. Thus the coefficient $B_{1}^{j}$ defines the force distribution on the array of cylinders at the trapped mode frequency. The expressions for $B_{1}^{j}$ have been defined by (2.24), (2.25) or (2.26), thus we know the force distributions as closed formulae.

We have compared the wave force distributions given by (2.25) with the wave force distributions induced in a long array of cylinders in the open sea at the nearresonant states, and found close similarities. Figures $4(a)$ and $4(b)$ show the wave force distributions along an array of 50 cylinders in the open sea at the near-resonant states, computed using the interaction theory of Linton \& Evans (1990), whereas figures $4(c)$ and $4(d)$ show wave force distributions for Dirichlet trapped mode waves for 50 cylinders across the tank. This illustrates the close similarities, although in the open-sea problem there exists energy dissipation to the far field; thus a slight phase shift occurs, whereas for the trapped mode there is no loss of energy and no phase shift.

Table 4 compares the Dirichlet trapped mode wavenumbers of the second highest modes $(q=N-1)$ in the channel with the wavenumber at which the peak load occurs within a finite array of $N$ cylinders in the open sea. For a sufficient number of cylinders they agree very well, which also shows the close similarities between these two cases.

In the open sea problem, there exist several near-resonant states below the wavenumber at which the highest wave force is observed below $k s=\pi / 2$. An example is shown in figure 4(b), where close similarities are found between the near-resonant state and the third highest trapped mode $(q=48)$. Such a mode-like behaviour of the near-resonant phenomenon in a long array of cylinders was first discovered by Maniar \& Newman and discussed in Newman (1997). Table 5 compares the wavenumbers at which such a near-resonant phenomenon occurs with the Dirichlet trapped mode wavenumbers. They agree very well, which also shows their resemblance.

\subsection{Analogy with a mass-spring oscillating system}

The wave force distributions shown in figure 4 and formulated in (2.24), (2.25) and (2.26) seem to resemble the modes of a mechanical vibration system. The physical 

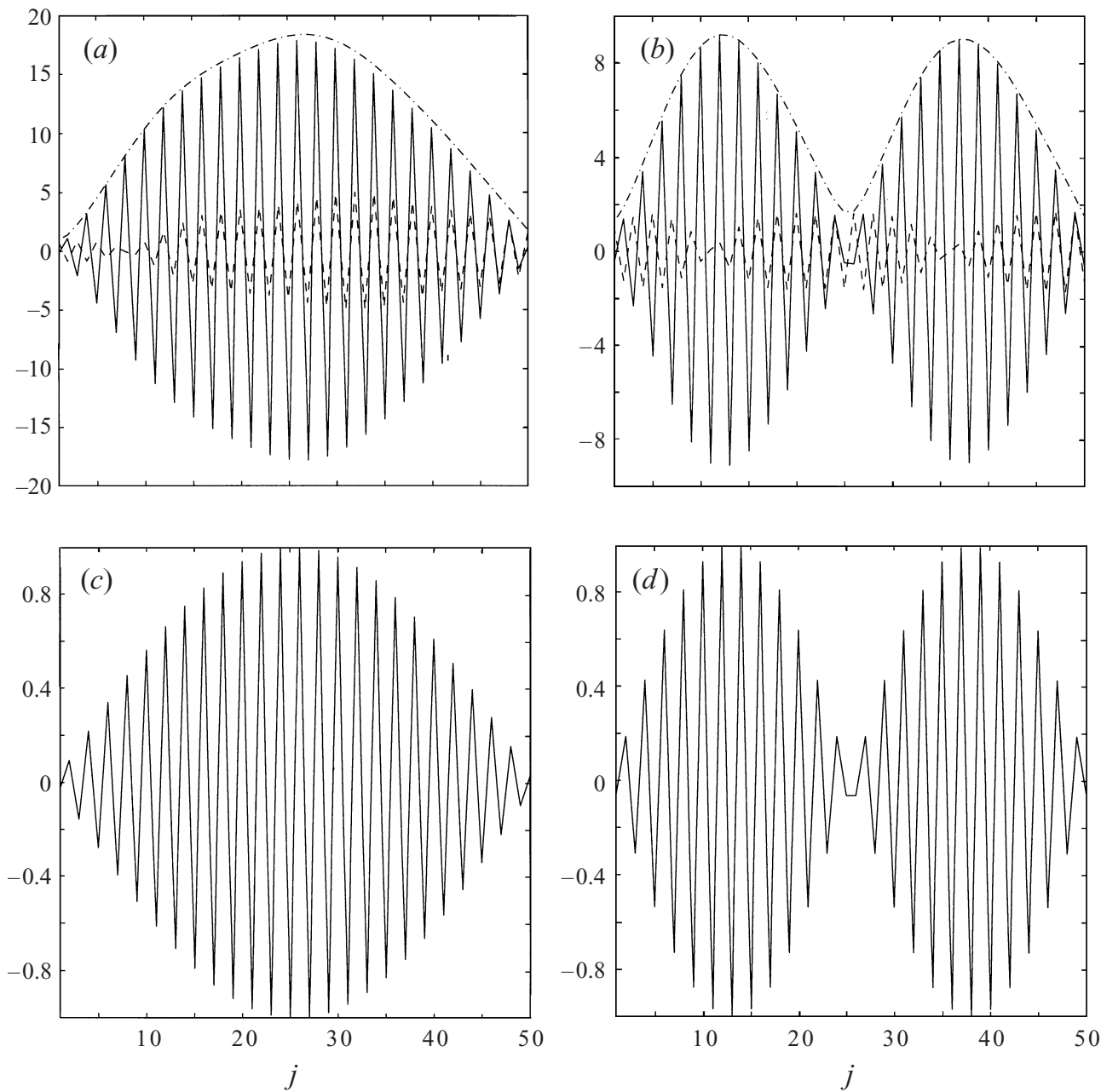

FiguRE 4. Comparison of the distribution of in-line wave forces along an array of 50 cylinders of $a / s=0.5$ in the open sea for the near-resonant state at $(a) k s=1.3889$ and $(b) k s=1.3819$, with the wave force distribution for Dirichlet trapped mode waves for $(c) q=49$ and $(d) q=48$. The lines in $(a)$ and $(b)$ indicate:,---- real part; - , imaginary part;.-- , magnitude.

background of the occurrence of the trapped modes around an array of cylinders may be closely related to the nature of a general periodic system. We have introduced the formulae (2.24), (2.25) and (2.26) above, and observed that they play a key role in constructing trapped modes around an array of cylinders. A complete physical understanding of the occurrence of such distribution functions, however, has not yet been achieved. Instead, we consider here a simple mechanical system which also has the same distribution functions (given by (2.24) and (2.25)) as the modal functions.

The basic unit in the mechanical analogy corresponds to one cylinder. It consists of a uniform massless cylindrical bar of unit cross-section, unit length and unit modulus of elasticity, with a unit point mass attached at its mid-length. The mass is allowed to oscillate in the direction of the axis of the bar. If the two free ends of the bar are fixed, the square of the natural frequency of vibration of the point mass is 4 , which may be considered to correspond to the Neumann trapped mode for one cylinder in the tank. 


$\begin{array}{crrcc} & N & q & k s & k_{p} s^{\dagger} \\ \text { (a) } & \infty & - & 1.55023 & 1.55023 \\ & 100 & 99 & 1.54446 & 1.54530 \\ & 50 & 49 & 1.53235 & 1.53799 \\ & 25 & 24 & 1.50796 & 1.52761 \\ & 10 & 9 & 1.41141 & 1.52162 \\ \text { (b) } & \infty & - & 1.39131 & 1.39131 \\ & 100 & 99 & 1.39071 & 1.39071 \\ & 50 & 49 & 1.38890 & 1.38891 \\ & 25 & 24 & 1.38182 & 1.38205 \\ & 10 & 9 & 1.33764 & 1.34635 \\ \text { (c) } & \infty & - & 1.32079 & 1.32079 \\ & 100 & 99 & 1.32041 & 1.32041 \\ & 50 & 49 & 1.31927 & 1.31925 \\ & 25 & 24 & 1.31474 & 1.31463 \\ & 10 & 9 & 1.28441 & 1.28518\end{array}$

TABLE 4. Comparison of the trapped mode wavenumber $k s$ for $q=N-1$ and $N_{F}=4$ with the wavenumber $k_{p} s$ at which the peak load occurs within a finite array of $N$ cylinders in the open sea ( $\dagger$ figures from table 3 of Maniar \& Newman 1997). The case for $N=\infty$ shows the Neumann trapped mode wavenumber for a single cylinder. (a) $a / s=0.2$, (b) $a / s=0.5$ and (c) $a / s=0.8$.

$\begin{array}{ccc}q & k s & k_{p} s \\ 49 & 1.38890 & 1.38891 \\ 48 & 1.38181 & 1.38187 \\ 47 & 1.37050 & 1.37066 \\ 46 & 1.35557 & 1.35560 \\ 45 & 1.33763 & 1.33820\end{array}$

TABLE 5. Comparison of the Dirichlet trapped mode wavenumber $k s$ for $N=50, a / s=0.5$ and $N_{F}=4$ with the wavenumber $k_{p} s$ at which the peak load occurs within a finite array of 50 cylinders in the open sea.

The basic unit is now replicated $N$ times in a straight line, by attaching the righthand end of one unit to the left-hand end of the neighbouring unit. Both ends are then fixed (fixed boundary condition) or allowed to be free to move (free boundary condition). In both cases, we can deduce the eigenvalues of the discrete system (omitting the rigid-body mode of the free boundary condition system) by the method of Faulkner \& Hong (1985) as

$$
\omega_{q}^{2}=2-2 \cos \left(\frac{q \pi}{N}\right), \quad \begin{cases}q=1,2, \ldots, N & \text { (for fixed boundary condition) } \\ q=1,2, \ldots, N-1 & \text { (for free boundary condition). }\end{cases}
$$

The corresponding eigenvectors giving the displacements are

$$
\begin{gathered}
d_{j}^{(q)}=\sin \frac{(2 j-1) q \pi}{2 N}, \quad q=1,2, \ldots, N \quad \text { (for fixed boundary condition), } \\
d_{j}^{(q)}=\cos \frac{(2 j-1) q \pi}{2 N}, \quad q=1,2, \ldots, N-1 \quad \text { (for free boundary condition), }
\end{gathered}
$$

where index $q$ designates the number of the mode, and the subscript $j$ identifies the mass. 
These are identical with the formulae for $B_{2 n+1}$ in (2.24) and (2.25). Thus (3.8) represents the wave force distributions for Neumann trapped modes, whereas (3.9) represents those for Dirichlet trapped modes. This analogy with the classical periodic structure, for which a rich literature is available, opens up several opportunities for the analysis of hydrodynamic resonances in periodic systems.

\section{Conclusions}

Trapped modes around a row of vertical circular cylinders in a wave channel which are aligned along the plane perpendicular to the channel walls have been established to exist. The cylinders are identical and equally spaced, and the spacing between adjacent cylinders is $2 s$ while that between the ends of the cylinders and the channel walls is $s$.

Multipole expansions have been employed to construct the trapped mode potentials. However, each multipole potential radiates standing waves, which do not vanish even at large distances $|x|$. In order to cancel out the standing waves at large values of $|x|$ and to construct the trapped mode solutions, we have introduced 'empirical' formulae for the coefficients of the multipoles, by which cancellation of the standing wave components has been made. By introduction of these formulae, the cutoff wavenumbers have been shown to become much higher than those in the original problem, and below the cutoff wavenumber of the modified problem, one trapped mode has been found. Because $N$ independent such modified problems, each having different cutoff wavenumbers, can be defined for an array of $N$ cylinders, and only one trapped mode has been found for each modified problem just below the cutoff wavenumber, we may reach the simple conclusion that ' $N$ trapped modes exist for an array composed of $N$ cylinders'.

We note, however, that because the formulae for the coefficients of the multipoles are only sufficient conditions to satisfy the boundary conditions at large distances $|x|$, we may still have additional trapped modes. Thus we have to change the arguments to 'at least $N$ trapped modes exist'. Moreover, as is already known, for a large radius single cylinder $(a / s \gtrsim 0.6789)$ in a tank the Dirichlet trapped mode does not exist. This still applies for an array of cylinders; thus for an array of cylinders whose radii are larger than $0.6789 \mathrm{~s}$, we have to change the argument to 'at least $N-1$ Dirichlet trapped modes exist if $a / s \gtrsim 0.6789$ applies'.

Rigorous mathematical proof of the existence of the trapped modes has not been made in this paper. The existence of the $N$ th trapped mode $(q=N)$ can be easily shown, however, by appropriately copying the trapped mode wave for the one cylinder case. For an obstacle which is symmetric about the centreline of the channel, the existence proof of the trapped mode has already been made by Evans et al. (1994); thus for an array of such obstacles, the $N$ th trapped mode has been shown to exist. For the other modes including the first mode, the existence proof remains to be resolved.

An analogy of the trapped modes with the near-resonant phenomenon in the wave diffraction around an array of cylinders has also been presented. By means of the analogy, mode-like behaviour in the near-resonant phenomenon has been explained in this paper. Another analogy has also been drawn with a mass-spring system as a classical periodic structure, by which we may obtain some physical insights to help understand the phenomenon. Porter \& Evans (1998) have discussed another analogy, between the near-resonant phenomenon in an array of cylinders and Rayleigh-Bloch waves. The latter exist at exactly the same wavenumbers as our results shown in 
table 4. Equivalency of the problem of Rayleigh-Bloch waves in a periodic cylinder array and the trapped modes around a row of cylinders in the wave channel can be anticipated, and it has been demonstrated very recently by Porter \& Evans (1999).

T.U. would like to acknowledge the support of the Kajima foundation during his one-year visit to the University of Oxford.

\section{REFERENCES}

Callan, M., Linton, C. M. \& Evans D. V. 1991 Trapped modes in two-dimensional waveguides. J. Fluid Mech. 229, 51-64.

Evans, D. V., Levitin, M. \& Vassiliev, D. 1994 Existence theorems for trapped modes. J. Fluid Mech. 261, 21-31.

Evans, D. V., Linton, C. M. \& Ursell, F. 1993 Trapped mode frequencies embedded in the continuous spectrum. Q. J. Mech. Appl. Maths 46, 253-274.

Evans, D. V. \& Porter, R. 1997 a Trapped modes about multiple cylinders in a channel. J. Fluid Mech. 339, 331-356.

Evans, D. V. \& PORTER, R. $1997 b$ Near-trapping of waves by circular arrays of vertical cylinders. Applied Ocean Research 19, 83-99.

Evans, D. V. \& Porter, R. 1998 Trapped modes embedded in the continuous spectrum. Q. J. Mech. Appl. Maths 52, 263-274.

FAulkner, M. G. \& Hong, D. P. 1985 Free vibrations of a mono-coupled periodic system. J. Sound Vib. 99, 29-42.

Gradshteyn, I. S. \& Ryzhik, I. M. 1994 Tables of Integrals, Series, and Products, 5th edn. Academic.

Linton, C. M. \& Evans, D. V. 1990 The interaction of waves with arrays of vertical circular cylinders. J. Fluid Mech. 215, 549-569.

Linton, C. M. \& Evans, D. V. 1992 The radiation and scattering of surface waves by a vertical circular cylinder in a channel. Phil. Trans. R. Soc. Lond. A 338, 325-357.

Linton, C. M. \& McIver, P. 1996 The scattering of water waves by an array of circular cylinders in a channel. J. Engng Maths 30, 661-682.

Maniar, H. D. \& Newman, J. N. 1997 Wave diffraction by a long array of cylinders. J. Fluid Mech. 339, 309-330.

MCIVER, M. \& Linton, C. M. 1995 On the non-existence of trapped modes in acoustic waveguides. Q. J. Mech. Appl. Maths 48, 543-555.

McIver, P. \& BenNetT, G. S. 1993 Scattering of water waves by axisymmetric bodies in a channel. J. Engng Maths 27, 1-29.

Newman, J. N. 1997 Resonant diffraction problems. Proc. 12th Intl. Workshop on Water Waves and Floating Bodies, pp. 307-308. Carry-le-Rouet, France.

Porter, R. \& Evans, D. V. 1998 Prediction of resonances due to waves interacting with finite linear arrays of cylinders. Proc. 13th Intl. Workshop on Water Waves and Floating Bodies, pp. 127-130. Alphen aan den Rijn, The Netherlands.

Porter, R. \& Evans, D. V. 1999 Rayleigh-Bloch surface waves along periodic gratings and their connection with trapped modes in waveguides. J. Fluid Mech. 386, 233-258.

Ursell, F. 1951 Trapping modes in the theory of surface waves. Proc. Camb. Phil. Soc. 47, 347-358.

UtsunomiYa, T. \& EATOCK TAYLOR, R. 1998 Analogies for resonances in wave diffraction problems. Proc. 13th Int. Workshop on Water Waves and Floating Bodies, pp. 159-162. Alphen aan den Rijn, The Netherlands. 